\title{
Horst Dieter Schlosser, Sprache unterm Hakenkreuz. Eine andere Geschichte des Nationalsozialismus
}

Köln : Böhlau, 2013, 423 p., 34,90€

Marcel Tambarin

\section{CpenEdition}

Journals

Édition électronique

URL : http://journals.openedition.org/ifha/7722

DOI : 10.4000/ifha. 7722

ISSN : 2198-8943

Éditeur

IFRA - Institut franco-allemand (sciences historiques et sociales)

Référence électronique

Marcel Tambarin, «Horst Dieter Schlosser, Sprache unterm Hakenkreuz. Eine andere Geschichte des Nationalsozialismus », Revue de l'IFHA [En ligne], Date de recension, mis en ligne le 15 décembre 2013, consulté le 22 septembre 2020. URL : http://journals.openedition.org/ifha/7722 ; DOI : https://doi.org/ 10.4000/ifha. 7722

Ce document a été généré automatiquement le 22 septembre 2020.

(CIFHA 


\title{
Horst Dieter Schlosser, Sprache unterm Hakenkreuz. Eine andere Geschichte des Nationalsozialismus
}

Köln : Böhlau, 2013, 423 p., 34,90€

\author{
Marcel Tambarin
}

Pour Horst Dieter Schlosser, philologue émérite de l'université de Francfort également connu pour avoir lancé l'initiative Unwort des Jahres - ce «non-mot» qui depuis 1991 prend le contre-pied critique du «mot de l'année » pour dénoncer les égarements du discours public -, la langue nazie ne représente pas plus un écart de langage que le nazisme ne constitue un accident de l'histoire.

C'est donc par la genèse de la langue nazie sous la république de Weimar, et même en remontant bien au-delà, par une rétrospective qui ramène au début du XIXème siècle, que s'ouvre cette étude de "la langue sous la croix gammée». Le premier chapitre rappelle ainsi que, sur le plan de la langue et de l'idéologie qu'elle véhicule, l'année 1933 n'a jamais constitué la césure pour laquelle elle est souvent présentée. La république de Weimar, mais aussi les régimes qui l'ont précédée, ont en effet fourni une grande partie du lexique comme de l'argumentaire nazis, tout particulièrement en ce qui concerne le discours antisémite, comme en témoigne une remarque incidente mais révélatrice de Thomas Mann dans un éloge de Gerhard Hauptmann prononcé en 1922. S'ensuivent des chapitres sur la langue du nazisme à ses débuts, sur la prise du pouvoir en 1933, sur les premières années du régime, et ainsi de suite jusqu'aux années de guerre et à l'effondrement de 1945. Seul le dernier des 17 chapitres, consacré à la langue de "l'autre Allemagne ", c'est-à-dire de la résistance au nazisme, échappe à cette progression strictement chronologique. Mais à sa manière, ce chapitre révèle lui aussi les limites d'une approche qui se veut exhaustive, puisqu'il se trouve éclaté en une multitude de sous-chapitres consacrés à toutes les résistances connues, des Églises aux communistes, de la Rose Blanche aux sociaux-démocrates, en passant par Stauffenberg et Goerdeler pour n'en citer que quelques exemples. A noter toutefois qu'au début de chaque chapitre figurent les titres et la pagination des sous-chapitres respectifs, ce qui 
facilite certes la lecture ciblée, mais ne remplace pas un index des mots-clés, qui aurait peut-être mis en évidence quelques répétitions, mais qui aurait surtout permis de tirer un meilleur profit d'un ouvrage dont la lecture cursive n'est pas toujours des plus agréables. Une brève introduction de 5 pages et une conclusion quant à elle substantielle de près de 13 pages encadrent l'ensemble, complété par la liste des sources et des références bibliographiques, et se terminant par un index des noms propres.

Ce découpage présente certes l'avantage de permettre une lecture dissociée de chacun de ces chapitres assez courts, d'une longueur variable de 6 à 40 pages, mais il a pour inconvénient qu'il ne permet pas vraiment de dégager d'idées-forces avant d'en arriver à la conclusion. Cette dernière, qui se présente comme un résumé de l'ouvrage, offre toutefois une liste des différents procédés employés par les nazis pour mettre la langue au pas et rappelle la méthode déjà mise en évidence par Victor Klemperer dans LTI pour en asseoir l'usage : la répétition et l'effet cumulatif provoqué par la répétition, auquel H. D. Schlosser ajoute la désambiguïsation, c'est-à-dire en l'occurrence la réduction sémantique d'un mot à une seule signification, qui aboutit à un appauvrissement de la langue également déjà observé par V. Klemperer dans son livre de 1947. L'ouvrage s'achève par des considérations quelque peu inattendues sur la RDA, dont la dictature communiste se résumerait à un simple " changement d'étiquette »alors qu'à l'opposé l'auteur n'explique pas pourquoi et comment l'Allemagne de l'Ouest a réussi à se débarrasser de l'idéologie nazie, même s'il reconnait que le processus a pris du temps et n'a du reste pas totalement abouti, comme en témoigne l'emploi persistant de slogans nazis dans certains milieux.

Le postulat du livre étant que la répression physique sur laquelle reposent les dictatures ne saurait suffire à les asseoir et que c'est à travers la mise au pas de la langue qu'elles s'exercent dans toute leur ampleur, H. D. Schlosser entreprend de passer au crible la langue allemande entre 1933 et 1945 pour mettre en évidence l'emprise de la propagande nazie, tantôt en commentant des termes ou expressions aussi connus que Kraft durch Freude ou Reichskristallnacht, tantôt en montrant à travers leur contextualisation comment des termes d'apparence banale aujourd'hui, comme anständig ou asozial, étaient pourtant le reflet, sinon l'instrument de l'idéologie nazie. C'est là toute la richesse du livre qui multiplie les exemples montrant à quel point le régime nazi a généralisé la manipulation de la langue, jusque dans le détail et souvent jusqu'à l'outrance, comme par exemple en abusant des composés du mot Volk, qui se retrouve dans tous les domaines, comme en attestent la Volkswagen et le Volksempfänger, mais aussi un Volkstraktor demeuré au stade de la conception. Comme cette mise au pas de la langue ne tolérait aucune divergence dans son emploi et qu'elle pénétrait la langue jusque dans son usage quotidien, il apparaît finalement, la bureaucratisation et la militarisation croissante de la société aidant, que même la langue utilisée en privé relevait plus de la langue $d u$ nazisme que de la langue sous le nazisme - exception faite des plaisanteries et des jeux de mots que l'ouvrage n'aborde toutefois qu'à la marge. Il s'avère d'ailleurs que dans certains cas l'usage s'est perpétué bien au-delà de l'effondrement du régime: parmi les exemples peut-être moins connus de cette entreprise d'ancrage linguistique de la dictature, H.D. Schlosser rappelle ainsi que l'alphabet téléphonique porte encore la marque des expurgations nazies qui firent disparaître David et Nathan, qui ne sont jamais revenus, à l'instar de beaucoup d'exilés ou de déportés qui portaient l'un de ces noms toujours remplacés aujourd'hui par Dora et Nordpol. De même le slogan Du bist Deutschland de la campagne de marketing social de 
2005 a-t-il déjà eu un précédent au milieu des années 1930, puisqu'avant d'interpeller les citoyens de la République fédérale il a déjà servi à encenser Hitler.

$\mathrm{Au}$ fil des chapitres et des exemples cités se dégagent les principaux procédés mis en oeuvre par le régime nazi pour assurer sa mainmise sur la langue : la mise en valeur systématique des termes-clés du régime national-socialiste et la dévalorisation, ou plutôt la disqualification tout aussi systématique des autres (comme " démocratie » ou « république »); la répétition, la redondance, voire le matraquage des termes les plus emblématiques de l'idéologie nazie ; la germanisation de la langue, en particulier dans le domaine des toponymes et des patronymes, mais aussi de la mode, jugée trop marquée par le français ; l'exacerbation de la langue mise au service de la guerre, pour ne pas dire sa militarisation même; et bien sûr le pendant de ce dernier procédé : l'euphémisation à des fins de dissimulation, pour camoufler les crimes du régime, comme les mesures d'euthanasie et surtout les exterminations, en particulier dans les camps. Le lecteur intéressé se prend toutefois souvent à regretter que la démarche choisie privilégie l'accumulation d'exemples au détriment d'une analyse plus fine des faits de langue et des faits de discours: s'il est facile et suffisant de signaler d'une phrase que la simple liste des Feuersprüche de l'autodafé de 1933 constitue à elle seule un véritable résumé de toute l'idéologie nazie, il aurait souvent été utile et instructif de détailler davantage les tenants et les aboutissant, comme l'auteur sait du reste le faire de manière éclairante, par exemple dans les passages consacrés au programme Kraft durch Freude.

Paradoxalement, le souci d'exhaustivité a pour corollaire que l'ouvrage reste souvent superficiel, car le souci de parcourir tous les champs empêche l'auteur de creuser et de montrer, sinon de démonter les mécanismes de manipulation de la langue. A cet égard, les apports du livre restent conditionnés par l'approche retenue qui mobilise finalement peu d'instruments méthodologiques pour se concentrer sur l'illustration par l'exemple, et il demeure donc de ce fait plus descriptif qu'explicatif. En forçant le trait, on pourrait dire qu'au final, «La langue sous la croix gammée» reste plus une histoire du national-socialisme que de la langue sous le national-socialisme. À cet égard d'ailleurs, le titre peut prêter à confusion, surtout si l'on néglige le sous-titre qui annonce bien quant à lui une "autre histoire du nazisme». Car il s'agit de fait d'une histoire du nazisme, non pas dans une approche proprement linguistique (ce qui en aurait sans doute réduit le public potentiel), mais considérée sous l'angle de l'utilisation que ce régime a fait de la langue à des fins de propagande - dont la langue elle-même ne constitue au demeurant qu'un des vecteurs indissociables des autres, comme le montrent les digressions sur la sculpture, les uniformes et la mise en scène... Somme toute, l'ouvrage possède les qualités de ses défauts : comme il s'agit d'un livre d'histoire en même temps que d'un livre sur la langue, le premier peut pallier l'éventuelle absence de connaissances qui empêcheraient de comprendre le second, en permettant de contextualiser les termes relevés et commentés. À ce titre, s'il n'apporte rien de vraiment nouveau au lecteur averti, il peut apporter d'autant plus aux lecteurs moins au fait de la période nazie, qui trouveront également dans une ample bibliographie de quoi prolonger leur lecture. 
INDEX

Index chronologique : Neuere und Neueste Geschichte

Thèmes : Politische Geschichte

\section{AUTEUR}

MARCEL TAMBARIN

Université de Bourgogne, Dijon 